\title{
E-cigarette, or vaping, product use associated lung injury (EVALI): new scenarios for physicians and radiologists
}

\author{
Carmine Guarino ${ }^{1}$, Ilaria Pedicelli ${ }^{1}$, Francesco Perna ${ }^{2}$, Valentina Di Spirito ${ }^{3}$, Giuseppe Fiorentino ${ }^{3}$, \\ Fabio Procaccini ${ }^{4}$, Gaetano Rea $^{5}$ \\ ${ }^{1}$ Unit of Bronchology, Monaldi Hospital, Azienda Ospedaliera dei Colli, Naples; ${ }^{2}$ Respiratory Medicine Division, \\ Department of Clinical Medicine and Surgery, Federico II University, Naples; ${ }^{3}$ Unit of Pathophysiology and \\ Respiratory Rehabilitation, Monaldi Hospital, Azienda Ospedaliera dei Colli, Naples; ${ }^{4}$ Department of Diagnostic \\ Imaging, Polydiagnostic Center Vega s.r.l., Naples; ${ }^{5}$ Department of Diagnostic Imaging, Monaldi Hospital, Azienda \\ Ospedaliera dei Colli, Naples, Italy
}

\begin{abstract}
A 59-year-old female ex-smoker with 40 pack year smoking history and a 5-year current e- cigarette (EC) use history, present-
\end{abstract}

Correspondence: Dr. Ilaria Pedicelli, Unit of Bronchology, Monaldi Hospital, Azienda Ospedaliera dei Colli, Via Leonardo Bianchi, 80131 Napoli, Italy.

Tel/Fax +39.081.7064141 - Mobile: +39.334.3225492.

E-mail: ilaria.pedicelli@gmail.com

Key words: E-cigarette; EVALI; organizing pneumonia; exogenous lipoid pneumonia; lipid laden macrophages.

Contributions: CG, design and revision of the work; IP, conception and design of the work, interpretation of data, drafting and revision of the work; FP, analysis and interpretation of data and revision of the work; VDS, GF, interpretation of data; FP, conception, design and revision of the work; GR, interpretation of data, drafting and critical revision of the work. All the authors have read and approved the final version of the manuscript and agreed to be accountable for all aspects of the work.

Conflict of interest: the authors declare no conflict of interest.

Funding: All the authors declare having not benefited from any source of funding for this work.

Informed consent: Consent was obtained from the patient for publication of this case report and accompanying images.

Received for publication: 3 June 2021.

Accepted for publication: 6 October 2021.

Publisher's note: All claims expressed in this article are solely those of the authors and do not necessarily represent those of their affiliated organizations, or those of the publisher, the editors and the reviewers. Any product that may be evaluated in this article or claim that may be made by its manufacturer is not guaranteed or endorsed by the publisher.

${ }^{\circ}$ Copyright: the Author(s), 2021

Licensee PAGEPress, Italy

Monaldi Archives for Chest Disease 2022; 92:1962

doi: 10.4081/monaldi.2021.1962

This article is distributed under the terms of the Creative Commons Attribution-NonCommercial International License (CC BY-NC 4.0) which permits any noncommercial use, distribution, and reproduction in any medium, provided the original author(s) and source are credited. ed with progressive dyspnea on exertion and daily cough for 2 months. A CT scan showed a consolidation area with air bronchogram in the middle lobe and non-calcific bilateral nodules, which could be attributed to community-acquired pneumonia. The patient was treated with empiric antibiotics and systemic steroids for 10 days. Infectious, neoplastic and autoimmune pathologies were excluded, whereas a broncho-alveolar lavage revealed an accumulation of lipids in the cytoplasm of the alveolar macrophages. Despite the recommendation of vaping cessation, the patient continued to use EC. A new CT exam, carried out after 18 months, showed reversed halo sign (RHS), patchy groundglass opacity (GGO), pleuro-parenchymal bands, and indeed perilobular pattern, suggestive of organizing pneumonia (OP). The final diagnosis was e-cigarette, or vaping, product use Associated Lung Injury (EVALI)- related OP.

\section{Introduction}

In recent years the increased number of hospitalizations for acute lung injury related to electronic cigarettes (ECs), in particular in the United States, has led to a growing scientific interest regarding "vaping" diseases [1]. Although many cases of acute respiratory disease and respiratory failure related to the use of ECs have been described, few cases of EC pulmonary damage with a subacute or chronic course are discussed [2-4]. Considering the increase in the use of ECs, the confusing effects of mild or nonspecific symptoms and the variability of chest images (which can be similar to other respiratory diseases), we believe that a higher percentage of the cases of e-cigarette, or vaping, product use associated lung injury (EVALI) is not recognized and that the pathology is still largely underestimated.

In the following case report about a five-year EC-user female patient, the radiological findings, the broncho-alveolar lavage (BAL), cytology and laboratory data highly indicated chronic EVALI related to organizing pneumonia (OP).

\section{Case Report}

A 59-year-old woman, with a 40 pack-year and a current 5-year e-cigarette smoking history was taken a high-resolution $\mathrm{CT}$ of the chest (HRCT) because of a two-month history of progressive dysp- 
nea and daily cough. The HRCT revealed the presence of a large consolidation with air bronchogram of the middle lobe and non-calcific bilateral nodules, in particular a $9 \mathrm{~mm}$ nodule in the apical segment of the right lower lobe and $7 \mathrm{~mm}$ in the same segment of the left lower lobe. Mild emphysematous changes in both upper lobes (Figure 1) were also visible. The patient was treated with ceftriaxone ( $2 \mathrm{~g}$ daily) for a presumed diagnosis of community-acquired pneumonia and systemic steroids for 10 days to cover a presumed COPD exacerbation. Due to the persistence of symptoms, the patient was admitted to our hospital. She reported to have taken a business trip to Romania about 4 months earlier, but she did not mention any other noteworthy pathologies. There were no significant occupational or domestic exposures (specifically to birds or other animals, or moulds), or hobbies of note. Upon physical examination, the auscultation of the chest revealed a basal bilateral

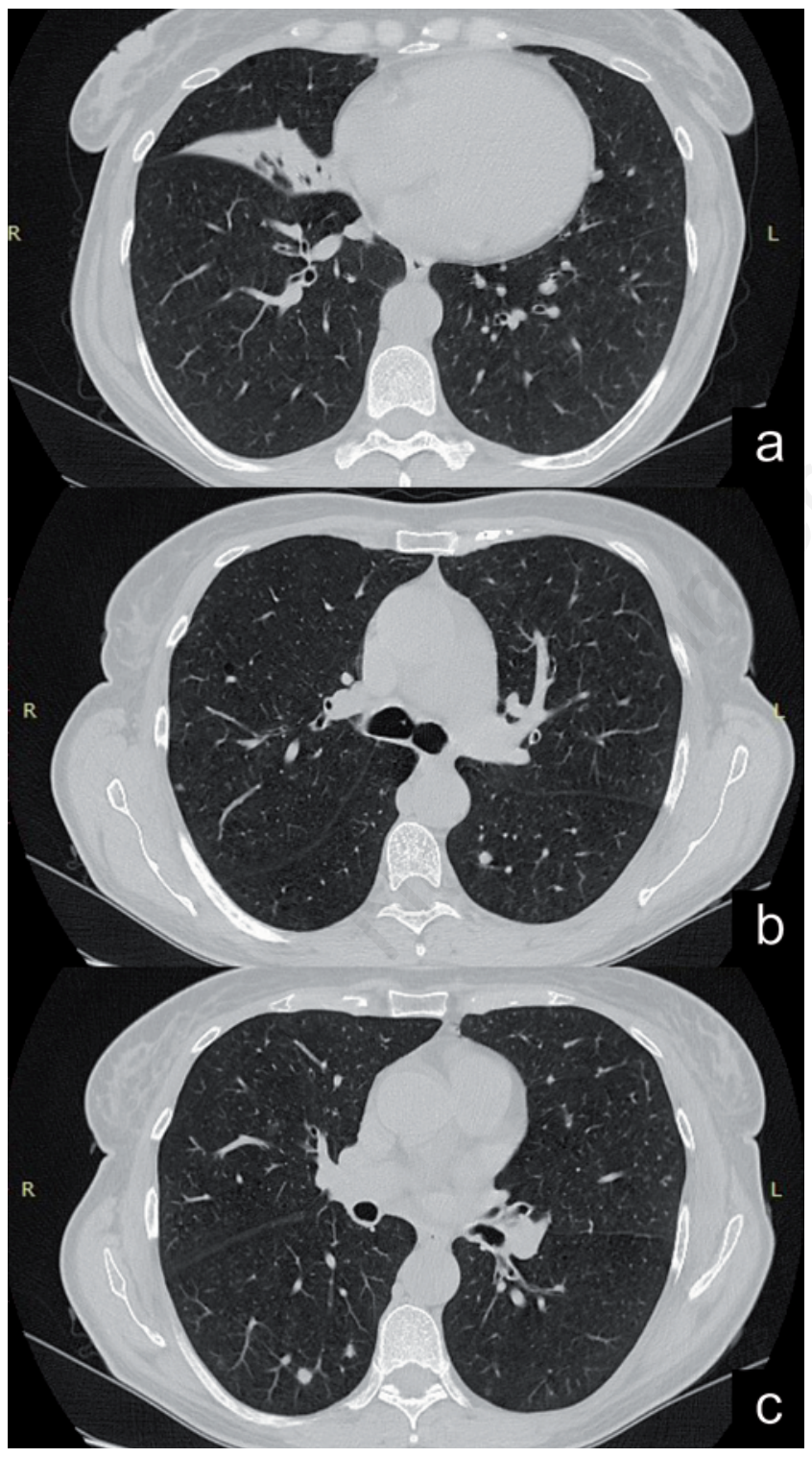

Figure 1. Chest HRCT: complete consolidation/atelectasis of the middle lobe with air bronchogram (a) non-calcific, indeterminate nodule in left lower lobe (b); non-calcific, indeterminate nodules in right lower lobe (c). reduction of breath sound with no crackles. The heart rate and blood pressure were normal; the oxygen saturation in the haemoglobin was 95\% under room air. Blood counts revealed an increased white blood cell (WBC) count of 11,820 cells $/ \mathrm{mL}$ (differential count: neutrophils $55.4 \%$, lymphocytes $36.3 \%$, monocytes $7.5 \%$, eosinophils $0.4 \%$, basophils $0.4 \%$ ). The erythrocyte sedimentation rate (ESR) was $17 \mathrm{~mm} / \mathrm{h}$ and C-reactive protein (CRP) was $0.4 \mathrm{mg} / \mathrm{dL}$.

The insidious onset of the disease and previous radiological data posed considerable doubts regarding potential differential diagnoses; therefore, further investigations were conducted. An 18 FDGPET showed a normal tracer uptake and serum tumor marker levels of CA15-3, CA19-9, CA 125, CEA, CYFRA 21-1 and NSE were in the normal range. Tests for influenza viruses, as well as urine antigen tests for legionella pneumophila, streptococcus pneumoniae, mycoplasma pneumoniae, and chlamydia pneumonia were negative. A search on sputum for mycobacterium tuberculosis (direct, culture, and PCR) and atypical mycobacteria were also negative. The autoimmune profile and antineutrophilic cytoplasmic antibodies were normal. A video-bronchoscopy with BAL was performed and it showed negative microbiology (fungal, viral, and bacterial pathogens were tested). Regarding the cytology of the BAL fluid, differential cell count showed 2\% lymphocytes, 3\% neutrophils, 95\% macrophages, and $0 \%$ eosinophils and revealed numerous large alveolar macrophages with similar-anthracotic pigment in their cytoplasm, Oil red $\mathrm{O}$ staining positive $(>50 \%)$ for extensive accumulation of lipid material (Figure 2). A spirometry revealed an obstructive ventilatory defect with $\mathrm{FEV} 1=1.39 \mathrm{~L}$ (61\% predicted), $\mathrm{FVC}=2.38 \mathrm{~L}$ ( $88 \%$ predicted), and $\mathrm{FEV}_{1} / \mathrm{FVC}=58.47 \%$. A more

careful evaluation of her anamnesis revealed that the patient has used electronic cigarettes in the last 5 years. She was advised to stop vaping immediately and prednisolone was prescribed for her $(25 \mathrm{mg}$ daily for 2 months). In addition, the patient was given an inhalation therapy with LABA/LAMA combination for stable COPD, which resulted in a substantial improvement in her symptoms. After one month of therapy, an HRCT of the chest revealed ground-glass opacities (GGO) diffuse to lower lobes with OP-like pattern (pleuroparenchymal bands, GGO and reversed halo sign), linear opacities, and GGO in the middle lobe and lingula (Figure 3a). Unfortunately, the patient continued to use ECs, despite the recommendation of vaping cessation. One last chest HRCT was performed eighteen months later, and it showed a pleuro- parenchymal band in the right lower lobe and GGO in the left lower lobe, which were a strong indication of sequelae of OP (Figure 3b). All data finally suggested a diagnosis of EVALI with OP dominant pattern with rare and sporadic small nodules of OP completely disappeared after steroid therapy, no macroscopic lung fat at HRCT and with a high concentration of lipid-laden macrophages (LLM) in BAL.

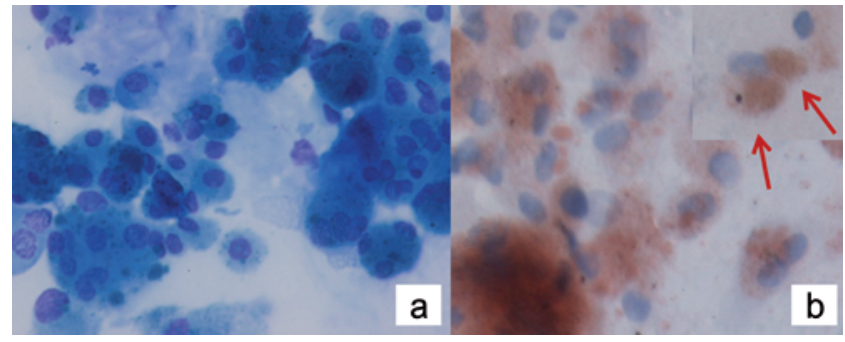

Figure 2. a) Large alveolar macrophages with anthracotic-like pigment in their cytoplasm (May Grunwald- Giemsa staining (400x magnification). b) Lipid-laden macrophages stained with Oil Red $O$ staining (400x magnification). 


\section{Discussion}

An electronic cigarette is a device designed to simulate and replace traditional smoking systems. It generally contains a mixture of ingredients (mainly nicotine, propylene glycol, vegetable glycerin, water, and flavoring agents), and is used to control nicotine addiction in order to promote its cessation.

In 2015, the use of ECs was highly concentrated in the US $(56 \%)$ and UK (12\%). However, the use of electronic vaping devices containing nicotine or tetrahydrocannabinol (THC, i.e., the main psychoactive constituent of cannabis), has greatly increased among young students in recent years. Its use has been associated with a growing number of cases of "vaping" related- lung diseases, represented by the acronym EVALI (e-cigarette or vaping product use- associated lung injury). An FDA and CDC document has reported a total of 2,668 hospitalized as EVALI cases between August 2019 to January 2020. In particular, 82\% of the patients used ECs containing THC, and 14\% used ECs containing exclusively nicotine [1]. The report also highlighted that the broncho- alveolar lavage fluid of EVALI patients showed the presence of Vitamin E acetate, which might have been used as an additive in ECs production or as a thickener in THC products. Vitamin E acetate impairs normal lung function $[5,6]$.

Diagnosing EVALI is a process based on exclusion. Use of ECs at least 90 days before symptoms appear, appropriate radiological images, and the absence of pulmonary infection on initial workup represent the CDC criteria for a "confirmed case of EVALI".

According to the imaging (CT-HRCT), the patterns of "vaping related" lung injury include OP, respiratory distress syndrome (RDS), diffuse alveolar damage (DAD), acute eosinophilic pneumonia, hypersensitivity pneumonitis (HP), exogenous lipoid pneumonia (ELP), and giant cell interstitial pneumonia [7,8]. OP is a reaction to an irritation caused by a trigger of any kind, and is characterized by patchy loose plugs and granulation tissue filling the lung alveoli and respiratory bronchioles [9]. OP can be idiopathic, in which case it is defined as cryptogenic organizing pneumonia (COP) and classified among idiopathic interstitial pneumonia, according to the 2002 ATS/ERS classification [10]. However, it can also be the consequence of a lung injury due to infection (bacteria, viruses, parasites, fungi), toxicity of drug as amiodarone, betablockers, carbamazepine, etc., inhalation of a pathogen (cocaine), inhalation of toxic gas, gastroesophageal reflux, connective tissue disorders (rheumatoid arthritis, systemic lupus erythematosus, polymyositis/dermatomyositis, polymyalgia rheumatica), organ

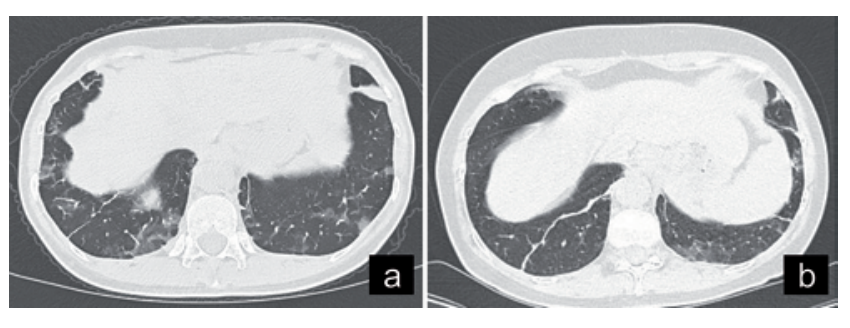

Figure 3. a) Chest HRCT of lower lobes after one month of steroid therapy showed RHS in right lower lobe, GGO areas and linear opacities in the lower lobes, highly suggestive of OP. b) Chest HRCT of lower lobes after 18 months showed mild focal GGO in left lower lobe, parenchymal bands and linear opacities in the lower lobes referred to sequelae of OP. transplant, or radiotherapy. In addition, OP can be histologically associated with pulmonary lesions of another nature such as vasculitis, lymphoma, lung cancer, hypersensitivity pneumonitis, eosinophilic pneumonia, acute interstitial pneumonia, non-specific interstitial pneumonia, or usual interstitial pneumonia $[11,12]$. Some cases of OP related to EC use have been described recently $[13,14]$.

From a clinical standpoint, OP can manifest itself with nonspecific symptoms, such as flulike illness, cough, dyspnea, fever, chest pain, sputum and hemoptysis. Bilateral patchy GGO, consolidations, or both, with peripheral or perilobular patterns represent typical CT/HRCT findings of OP. Most patients respond rapidly to corticosteroid therapy. Furthermore, the classification of OP as cryptogenic rather than secondary is clinically important, as the management of secondary OP also requires treatment of the underlying disease (eg, infection and connective tissue disease) and the avoidance of the potential offending agent (eg, medication and radiation injury) [15]. Previous reports and clinical experience suggested that secondary OP could be associated with a worse prognosis and less responsive to steroid treatment when compared with COP. Although in rare cases, the disease might also present a rapidly progressive respiratory failure picture and frequent relapses.

In our reported case, three elements suggested a direct relationship between pneumonia and the chronic irritating stimulus caused by the oily irritants in ECs: the inflammatory response (which persisted over time), the radiological findings (classically referable to OP in evolution), and the absence of typical macroscopic radiological evidence of lipoid pneumonia, despite the presence of LLM in BAL cytology samples.

Recently, there has been an increased interest related to the potential role of LLM in the detection of vaping-associated pulmonary injury (VAPI); particularly, Shields et al. have demonstrated the presence of LLM in $96 \%$ of healthy smokers and $58 \%$ of EC users, and almost subjects who have never smoked [16]. This evidence suggests that LLM in BAL has poor specificity and cannot be considered as a laboratory marker for EVALI [17]. The study also showed that EC users, positive to LLM, had high levels of cytokines IL 4 and IL10, which were absent in the airways of healthy smokers. These findings suggest that the increase of those cytokines in EC users could be the result of a compensatory response to impaired pulmonary hemostasis or glandular hypersecretion, characteristic to EC exposure. More specifically, IL-4 is an important pro-inflammatory modulator that stimulates the type 2 inflammatory response of Thelper cells and is directly involved in the hypersecretion of mucus [18]. IL10 has well-documented immunosuppressive properties through inhibition of the Th1-mediated immune response. Some studies also report the production of IL-10 by airway macrophages [19]. It is probable that the exposure to $\mathrm{EC}$, which promotes an inflammatory process on one hand, stimulates a greater expression of anti-inflammatory factors to balance its effects on the other hand.

In our case, the patient had been a heavy cigarette smoker for 40 years and has recently used EC for about 5 years. Precisely, she reported to have been using an OVALE brand mod box device, with a mixture of liquids consisting of nicotine (at the concentration of $20 \mathrm{mg} / \mathrm{mL}$ ), propylene glycol and vegetable glycerin (5050) and, or, tobacco flavor every day for about 8 hours a day. Therefore, it is certain that the increase in LLM component and the evidence of anthracotic pigments in alveolar macrophages is partly due to her history of exposure to cigarette smoke [20]. However, the clinical and radiological manifestations (OP-like consolidation, nodules, and GGO) appeared only after prolonged exposure to EC, in absence of microbiological or autoimmune features that could 
have justified the appearance of the symptoms. Moreover, the improvement after treatment with systemic steroids seems to confirm the diagnosis of dominant EVALI-related OP-pattern. Many case reports have shown improvement with the use of corticosteroids, given their anti-inflammatory nature, but further research is needed to determine the ideal dosage and duration of treatment.

\section{Conclusions}

Despite the growing scientific interest in recent years about the use of ECs, there are still doubts about the role of alveolar macrophages in the genesis of respiratory disorders and the longterm damages caused by ECs. In patients who have had a prolonged use of ECs, a diagnosis of EVALI must always be suspected in cases of non-resolving radiological findings (like consolidations without macroscopic fat, which would make the diagnosis of lipoid pneumonia easy) after prolonged antibiotic therapy, or when GGO, nodules, or OP pattern are evident on HRCT exams.

\section{References}

1. Krishnasamy VP, Hallowell BD, Ko JY, et al. Update: Characteristics of a nationwide outbreak of e-cigarette, or vaping, product use-associated lung injury - the United States, August 2019-January 2020. MMWR Morb Mortal Wkly Rep 2020;24;69:90-4.

2. Viswam D, Trotter S, Burge PS, Walters GI. Respiratory failure caused by lipoid pneumonia from vaping e-cigarettes. BMJ Case Rep 2018;6;2018:bcr2018224350.

3. Fryman C, Lou B, Weber AG, et al. Acute respiratory failure associated with vaping. Chest 2020;157:e63-8.

4. Rajagopala S, Selvam N. An unusual case of respiratory distress. J Postgrade Med 2019;65:38-40.

5. Blount BC, Karwowski MP, Shields PG, et al. Vitamin e acetate in bronchoalveolar-lavage fluid associated with EVALI. N Engl J Med 2020;20;382:697-705.

6. Blount BC, Karwowski MP, Morel-Espinosa M, et al. Evaluation of bronchoalveolar lavage fluid from patients in an outbreak of e-cigarette, or vaping, product use-associated lung injury - 10 States, August-October 2019. MMWR Morb Mortal Wkly Rep 2019;15;68:1040-104.

7. Kligerman S, Raptis C, Larsen B et al. Radiologic, pathologic, clinical, and physiologic findings of electronic cigarette or vaping product use-associated lung injury (EVALI): Evolving knowledge and remaining questions. Radiology 2020; 294:491-505.

8. Henry TS, Kligerman SJ, Raptis CA et al. Imaging findings of vaping-associated lung injury. AJR Am J Roentgenol 2020;214:498-505.

9. Epler GR. Bronchiolitis obliterans organizing pneumonia. Arch Intern Med 2001;161:158- 64.

10. American Thoracic Society, European Respiratory Society. American Thoracic Society/European Respiratory Society international multidisciplinary consensus classification of the idiopathic interstitial pneumonias. Am J Respir Crit Care Med 2002; $15 ; 165: 277-304$.

11. Drakopanagiotakis F, Paschalaki K, Abu-Hijleh M, et al. Cryptogenic and secondary organizing pneumonia: clinical presentation, radiographic findings, treatment response, and prognosis. Chest 2011;139:893-900.

12. Baque-Juston M, Pellegrin A, Leroy S, et al. Organizing pneumonia: what is it? A conceptual approach and pictorial review. Diagn Interv Imaging 2014;95:771-7.

13. Khan MS, Khateeb F, Akhtar J, et al. Organizing pneumonia related to electronic cigarette use: A case report and review of literature. Clin Respir J 2018;12:1295-9.

14. Jankharia B, Rajan S, Angirish B. Vaping associated lung injury (EVALI) as an organizing pneumonia pattern - A case report. Lung India 2020;37:533-5.

15. Basarakodu KR, Aronow WS, Nair CK, et al. Differences in treatment and in outcomes between idiopathic and secondary forms of organizing pneumonia. Am J Ther 2007;14:422-6.

16. Shields PG, Song MA, Freudenheim JL, et al. Lipid laden macrophages and electronic cigarettes in healthy adults. EBioMedicine 2020;60:102982.

17. Butt YM, Smith ML, Tazelaar HD, et al. Pathology of vapingassociated lung injury. N Engl J Med 2019;381:1780-1.

18. Steinke JW, Borish L. Th2 cytokines and asthma. Interleukin4: its role in the pathogenesis of asthma, and targeting it for asthma treatment with interleukin-4 receptor antagonists. Respir Res 2001;2:66-70.

19. Branchett WJ, Lloyd CM. Regulatory cytokine function in the respiratory tract. Mucosal Immunol 2019;12:589-600.

20. Hansen MJ, Chan SP, Langenbach SY, et al. IL-17A and serum amyloid A are elevated in a cigarette smoke cessation model associated with the persistence of pigmented macrophages, neutrophils and activated NK cells. PLoS One 2014;18;9: e113180 